\title{
TWO CASES OF EPITHELIAL IMPLANTATION CYST OF THE IRIS
}

BY

\author{
R. Foster MOORe
}

LONDON

THE following are two cases of epithelial implantation cyst on the iris which have come under my care and upon which I have operated.

The first case has already been reported for me in its earlier stages by Mr. R. C. Davenport in the St. Bartholomew's Reports, 1924, Vol. LVII. p. 141. It may now be considered to be complete and so is worth reporting fully.

On May 17, 1922, a house surgeon wearing concave glasses was playing fives when the ball came back and broke the spectacle glass into his right eye. He was at once admitted into the hospital and I saw him the same evening. There was a clean linear wound through the whole thickness of the cornea extending completely from one side to the other, from about 10 o'clock to 4 o'clock and cutting across the middle of the cornea. The anterior chamber had already partly reformed, there was no prolapse of the iris, the pupil was circular, the lens had escaped damage and it was clear that no operative procedure was desirable.

Next morning the fundus was clearly seen and was normal, the eye was soft and on the front of the iris at about 4 o'clock at the site at which the cyst afterwards formed, was a small greyish white, rather woolly-looking mass. Examination both by X-rays and by the giant magnet gave a negative result, and transillumination of the globe produced no information.

The eye quickly healed and soon was nearly white. Sixteen days later the small mass on the front of the iris, already referred to, was smaller but was still clearly visible. A fortnight later a few very fine keratic deposits were present and the small mass on the iris could no longer be seen.

From this time the eye gradually settled down, the acuity with myopic correction became a complete $6 / 6$, but the eye was still for many weeks a little softer than the other one and was inclined to flush rather easily on examination. I many times expressed the feeling that there must be something, perhaps of the nature of some impalpable dirt carried in at the time of the accident that was irritating the eye and that was responsible for the softness, slight as it was, and the tendency to flush.

Ten months later the patient himself noticed a change in the eye. At the inner end of the wound behind the nasal end of the scar was a small spherical pearly cyst, and lying across it with its 


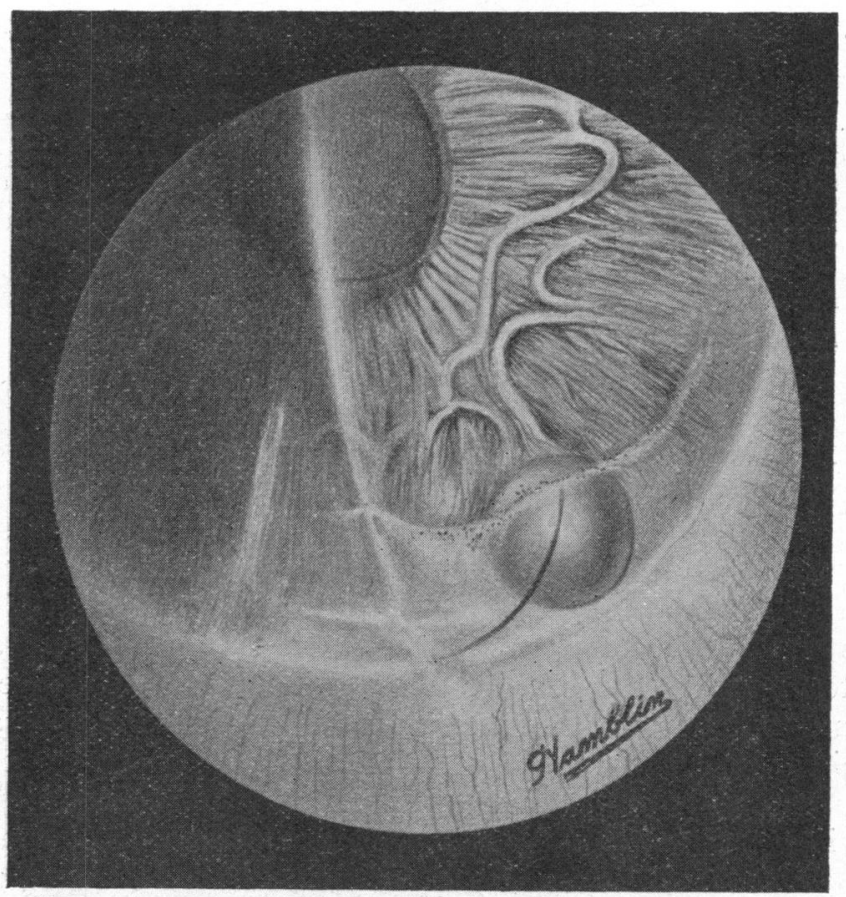

Plate 1.

root uppermost was an eyelash. It now seemed clear that this lash, owing to its curve, had lain completely hidden in the angle of the anterior chamber and that its presence was responsible for the slight softness of the eye and its irritability in the earlier stages; the growth of the cyst had pushed the lash forward and so brought it into view.

The cyst increased in size under observation, it was decided therefore to remove it. On July 27, 1923, that is 14 months after the original injury, a section was made with a keratome at the corneo-scleral junction at 5 o'clock, the lash was removed by means of an iris hook; it came out entirely free and was quite unattached to the cyst. The wound was now enlarged by a narrow von Graefe's knife, an iridectomy so planned as to include the cyst was then performed and the eye closed down. Sections of the cyst showed it was lined by squamous epithelium and was similar in all essentials to the cyst in Case II, Figs. 1 and 2.

Seeing that the lash had lain incubating, so to speak, in the anterior chamber for so long as 14 months, one wondered whether it would show evidence of growth. It was examined for me by Dr. R. G. Canti with respect to this point and he was unable to find any evidence that growth of it or of its root had occurred. Healing was uneventful, and full vision was again obtained. 
Five months later, that is in January, 1924, a year and eight months after the accident a little discomfort was again felt and a small cyst precisely similar to the former one and having exactly the appearance of a pearl, both in shape and sheen was seen far back in the angle of the anterior chamber; it was situated exactly in the coloboma as if in connection with the cut root of the iris, or ciliary body. It was small and by means of the slit-lamp it could be seen that its posterior surface was flattened by contact with the edge of the lens.

At this time Mr. J. H. Fisher was good enough to see him with me and it was agreed that removal was necessary. It was quite clear at the same time that being so far back it would be a matter of some delicacy to remove it. Operation was delayed for a fortnight as staphylococcus aureus was found to be present and during this fortnight the cyst increased in size. On February 26, 1924 , a general anaesthetic was administered by Dr. C. F. Hadfield, a wide flap of conjunctiva was raised from the nasal side and was dissected back to the limbus. A section was now made behind this flap with a small cataract knife and an endeavour was made to avoid transfixion of the cyst on passing the knife across the anterior chamber by working round it on its nasal side.

Having completed the section the conjunctival flap was pulled upon; this made the wound gape and caused the cyst to present in the wound. It was then dissected round by means of an iris repositor and although it was ruptured during this manoeuvre it was completely removed. The ciliary processes were clearly exposed during the operation. The eye made a complete and uneventful recovery and now, April, 1930-that is seven years and eleven months after the accident his visual acuity is better than $6 / 6$ and the eye causes no trouble.

\section{Case II}

On December 11, 1929, a woman aged 48 years, was breaking some coal with a coal-hammer when something flew into her right eye. Five days later she noticed a spot in the eye and it became a little bloodshot and ten days after the accident she consulted me at the Moorfields Eye Hospital.

The scar of a perforating wound of the cornea was present, adjoining the limbus at 6 o'clock. A small whitish mass was visible on the front of the iris rather to the nasal side of the wound. The eye was little inflamed, the fundus appeared normal and the visual acuity was 6/9. The left eye was normal and healthy. Examination by the giant magnet was negative. On December 21, Mr. Bruce Hamilton, Senior House Surgeon to the hospital, made a keratome section below and removed the foreign body with iris forceps. It proved to be a small fragment of coal 


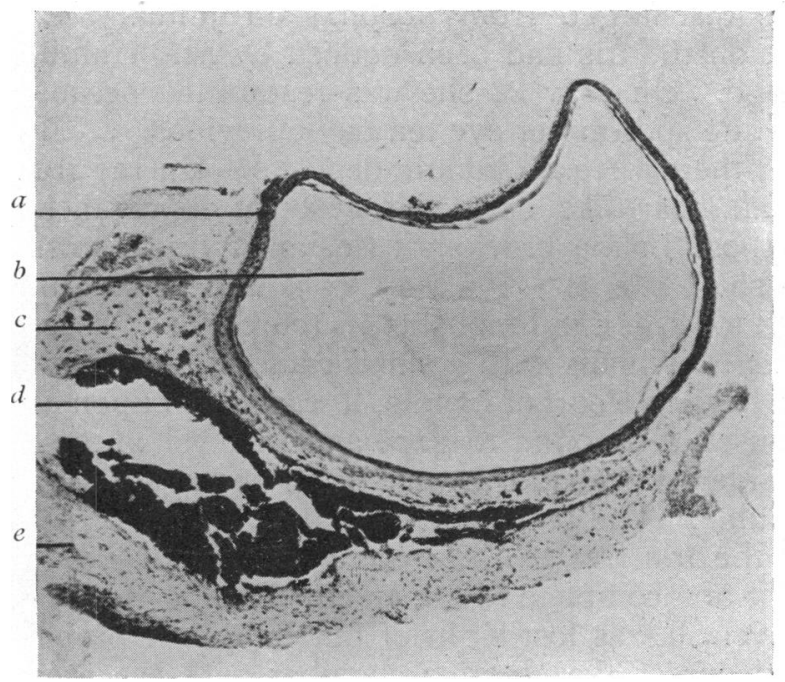

FIG. 1.

IMPLANTATION CYST OF IRIS. Low power view.

$a$ Cyst wall. $\quad b$ Keratinized contents of the cyst. $c$ Iris stroma. $d$ Pars iridica retinae. $e$ Remainder of the iris folded back upon itself.

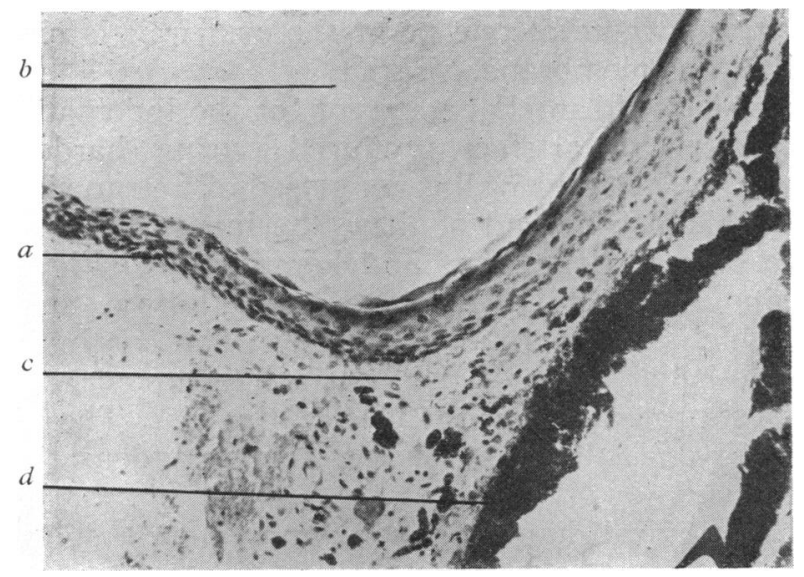

Fig. 2.

Implantation Cyst of Iris. High power.

$a$ Cyst wall. $b$ Keratinized cyst contents. $c$ Iris stroma. $d$ Pars iridica retinae. 
surrounded by exudate. The eye healed satisfactorily and the patient was discharged from hospital on January 4. A small woolly spot on the iris had been noticed by Mr. Hamilton before her discharge. On May 22 she was readmitted because she had noticed a white spot in her eye ten days previously. The eye was quite white, there were no keratic deposits. On the iris could be seen a small pearrl-like epithelial cyst in appearance precisely similar to those in the previous case, and lying across it was a small eyelash (Plate 1). On May 24 a flap of conjunctiva was turned back towards the limbus as in the previous case, a section of the cornea was made with a small cataract knife and using the conjunctival flap as a sort of handle, it was pulled upon as in Case I and this caused the wound to gape and the cyst to prolapse quite cleanly outside the eyeball. It was cut off and again the eye healed without trouble. The patient left the hospital on June 3 with the eye white, the iris free of the wound, no keratic deposits and a visual acuity of a complete $6 / 6$ unaided. During the manipulation the small eyelash was lost sight of but I think it probable that it was washed out of the anterior chamber with the escape of the aqueous ; it was certainly not attached in any way to the cyst.

Mr. H. B. Stallard was good enough to prepare sections for me and has supplied the following report:-

"The specimen was fixed in Zenker's solution, embedded in paraffin, and serial sections were cut and stained with haematoxylin and eosin.

" There is a cyst embedded in the iris. It projects forwards above the level of the anterior surface of the iris and backwards into the stroma, where at one point the cyst wall is close to the posterior limiting membrane. Fig. 1.

" The cyst is ovoid in shape, except for the indentation on the anterior surface, an artefact produced during hardening and section-cutting. The cyst wall is composed of four to six layers of stratified epithelial cells and of these the innermost two or three layers have become degenerate and keratinized. (Fig. 2). The cyst contains kerato-hyaline, an opaque, horny, structureless substance which stains pink with eosin. The continuitv of the anterior endothelial layer of the iris is interrupted at the place where the cyst projects above the level of the iris. The iris stroma in the vicinity of the cyst shows some condensation of its connective tissue.

"The pathological diagnosis is an implantation epithelial cyst of the iris."

The points of particular interest in these cases seem to be as follows :- In Case I it was remarkable that a clean penetrating cut should be made completely from one side of the cornea to the other without damaging the iris or the lens or without the iris 
becoming in any way adherent to the wound. The slight irritability and lowered tension of the eye made it seem certain that some foreign body had been carried into the eye, although no sign of it was discoverable after repeated examination, and it was only months afterwards when the cyst appeared pushing forward the eyelash that this judgment was justified. The lash was quite unattached to the cyst and the same was true of the second case, nor did it show any signs of growth although it had, so to speak, been incubated for several months. The method of exposure gave excellent results, it allowed one to get far back in the anterior chamber, especially in the instance of the second cyst in Case I and the slight distortion of the globe and gaping of the wound which could be produced by pulling on the conjunctiva using it as a handle caused the cysts to protrude prominently from the wound so that they were easily removed. The second cyst in Case I was placed so far back that the ciliary processes were clearly seen during operation and the cyst itself was flattened by contact with the edge of the lens. During the removal of this, its walls were lacerated and its contents escaped in part into the anterior chamber. Having regard to the nature of the contents, one would not expect any particular trouble from it nor has any arisen for so long a period of six years. Whether implantation cysts of this nature ever occur apart from the inclusion of an eyelash, I do not know but if so, they must be exceedingly rare.

There is one other point of possible interest, that in each case a small woolly-looking fragment was noticed on the front of the iris which afterwards seemed to disappear, as if in some way it were a precursor of the development of the cyst.

\section{THE NON-MAGNETISABLE METALLIC FOREIGN BODIES OF THE CORNEA, WITH THE REPORT OF A CASE}

BY

\section{B. W. RYCROFT}

BRADFORD

Metallic foreign bodies of the cornea may be arbitrarily divided into two classes based on their magnetic properties and it is with the non-magnetic and more uncommon type that I wish to deal, for the treatment of cases in this class may be relatively simple or extremely difficult.

Representative metals of this class are brass, copper, lead, solder and gold; all of these are found as foreign bodies on the cornea. 